\title{
Theoretical expectations for dark matter detection at the LHC
}

\author{
Howard BAER* \\ Dep't of Physics and Astronomy \\ University of Oklahoma \\ Norman, OK, 73019, USA \\ E-mail: baer@nhn. ou . edu
}

I present an overview of theoretical expectations for detection of dark matter (DM) at the LHC, concentrating entirely on supersymmetric candidates. En route, I present a unified theory which explains dark matter signals at indirect and direct detection experiments. While direct DM detection is likely impossible at LHC, detection of SUSY matter states is robust, where LHC with $\sqrt{s}=7 \mathrm{TeV}$ and $1 \mathrm{fb}^{-1}$ can access $m_{\tilde{g}} \sim 1 \mathrm{TeV}$ for $m_{\tilde{q}} \sim m_{\tilde{g}}$, and $m_{\tilde{g}} \sim 640 \mathrm{GeV}$ for $m_{\tilde{q}} \gg m_{\tilde{g}}$. Models with well-tempered neutralinos will soon be tested by Xenon-100/LUX, and should provide a distinctive mass edge at LHC in the $m\left(\ell^{+} \ell^{-}\right)$distribution. In the case of SUSY, neutralino dark matter now seems highly disfavored by both the magnitude of the dark matter density, and also the gravitino problem. Alternatively, the PQMSSM yields a solution to the strong CP problem, provides all the benefits of SUSY, and solves several cosmological problems. Predictions for SUSY particles at the LHC from the PQMSSM are quite different from the case of neutralino dark matter. Compelling models such as Yukawa-unifed SUSY or Effective SUSY, which would be excluded with neutralino DM, are perfectly viable with mixed axion/axino DM.

Identification of Dark Matter 2010

July 26-30, 2010

University of Montpellier 2, Montpellier France

\footnotetext{
${ }^{*}$ Speaker.
} 


\section{Introduction}

The astrophysical evidence for the existence of dark matter in the universe is now overwhelming, and comes from numerous disparate sources. Already, we know many of the properties of the putative dark matter particle(s): it must be massive, electrically neutral, and predominantly cold (non-relativistic). Of all the particle states in the Standard Model (SM), only neutrinos seem to have the first two of these properties. However, neutrinos constitute hot dark matter, so some other matter state is needed: the existence of dark matter requires new physics beyond that of the Standard Model.

In the theory literature, there exist numerous candidate states that might make up the dark matter: Kaluza-Klein (KK) photons or KK gravitons, lightest $T$-parity odd particles from Little Higgs theories, branons, Q-balls etc. However, dark matter emerges naturally from two quite different theories which solve long standing problems in particle physics.

The first of these, supersymmetry or SUSY[1], has been invoked to stabilize the hierarchy problem wherein scalar masses such as for the Higgs boson tends to blow up to the largest scale present in the theory. When the SM is supersymmetrized, the quadratic divergences are tamed; The resulting softly broken theory, the MSSM, provides a bonafide WIMP candidate- the lightest neutralino $\widetilde{Z}_{1}$. If local SUSY, or supergravity (SUGRA) is invoked, then the gravitino may also play the role of dark matter.

The other compelling theory is the Peccei-Quinn-Weinberg-Wilczek (PQWW) solution to the strong $C P$ problem[2]. t'Hooft's solution to the QCD $U(1)_{A}$ problem suggests that the QCD Lagrangian should contain a $C P$ violating $\frac{\bar{\theta}}{32 \pi^{2}} F \tilde{F}$ term, which leads to large contributions to the neutron EDM. But experiment tells us that $\bar{\theta}<10^{-11}$ [3]. PQ suggested an additional broken $U(1)_{P Q}$ global symmetry. The resulting Goldstone boson- the axion- provides additional field dependent $F \tilde{F}$ terms in the QCD Lagrangian. The axion field relaxes to the minimum of its potential, causing the entire $F \tilde{F}$ term to dynamically go to zero. In the process, coherent oscillations of the axion field fill the universe with non-relativistic axions, which are excellent candidates for CDM[4].

Of course, these two compelling theories- SUSY and PQWW- are not mutually exclusive, and actually enhance each other: one can build a PQMSSM theory[5] which may contain axions $a$ as well as $R$-parity odd axinos $\tilde{a}$. Thus, dark matter in the PQMSSM is not just one particle; one may have mixed axion-axino CDM, mixed axion-gravitino CDM or mixed axion-neutralino CDM.

As we will see, there are dark clouds on the horizon for theories with pure neutralino or pure gravitino CDM. However, mixed axion-ino CDM seems to work just fine! This has rather large implications for what LHC and dark matter detection experiments may find.

\section{SUSY at the LHC}

Due to time limits, I will focus here on gravity-mediated SUSY breaking models (SUGRA), wherein the gravitino is expected to be around the weak scale, and to set the mass scale for the other SUSY particles. Gauge mediation (GMSB) doesn't seem to naturally yield CDM. Anomaly mediation (AMSB) can yield the correct CDM abundance: for a recent analysis, see [6].

The paradigm model for SUSY phenomenology is the minimal supergravity or mSUGRA model[7] (also called the CMSSM by some authors). The mSUGRA model features the well- 
known parameter space

$$
m_{0}, m_{1 / 2}, A_{0}, \tan \beta, \operatorname{sign}(\mu) .
$$

One may stipulate the GUT scale parameters $m_{0}, m_{1 / 2}, A_{0}$ and $B_{0}$, and run them via RGEs down to the weak scale, and calculate all sparticle masses and mixings. The value of $B$ is traded for $\tan \beta$ upon scalar potential minimization. We adopt the Isajet subprogram Isasugra[8] for this purpose. Once the physical masses and couplings are known, decay widths and production cross sections may be calculated. These are all encoded in Isajet[9]. The neutralino relic density is calculated with IsaReD[10].

Assuming neutralino dark matter, one may calculate $p p \rightarrow \widetilde{Z}_{1} \widetilde{Z}_{1}$ production. However, there is nothing in the final state for detectors to trigger on, unless a hard QCD jet is radiated from the initial state. Thus, direct dark matter production is quite useless at LHC. Instead, for SUSY theories, one wants to produce the other matter states associated with the new physics, which may decay later into DM states. For LHC-a hadron collider- the strongly interacting states- the squarks and gluinos- usually have the largest cross sections. These are frequently also the heaviest states, so they are expected to decay via a cascade[11] into the lightest SUSY particle (LSP) plus a variety of hard jets and hard (isolated) leptons[12].

LHC has turned on at $\sqrt{s}=7 \mathrm{TeV}$ in 2010, and will continue running at this energy in 2011. Total SUSY production cross sections at $\sqrt{s}=7 \mathrm{TeV}$ are shown in Ref. [13]. In the case where $m_{\tilde{q}} \sim m_{\tilde{g}}$, these cross sections range to well over $10^{4} \mathrm{fb}$ for $m_{\tilde{g}} \sim 400-500 \mathrm{GeV}$. As of December, 2010, Atlas and CMS each have $\sim 0.045 \mathrm{fb}^{-1}$ of integrated luminosity, so there already could be hundreds of SUSY events lurking in their data!

The $\tilde{g}$ and $\tilde{q}$ decay usually through a cascade involving several steps into jets, leptons plus $E_{T}^{\text {miss }}$. Combining distinct sparticle production cross sections with the many decay possibilities yields of order $10^{5}$ distinct $2 \rightarrow n$ subproceses. The way to make sense out of these is to embed them into an event generator program, so the expected SUSY events can be generated probabilistically, and including QCD radiation, hadronization, and treatment of the underlying event.

We break the expected signatures up according to the presence of isolated leptons: jets $+E_{T}^{\text {miss }}$, $1 \ell+$ jets $+E_{T}^{\text {miss }}$, opposite sign dileptons (OS) plus jets $+E_{T}^{\text {miss }}$, same-sign dileptons plus jets $+E_{T}^{\text {miss }}$, $3 \ell$ plus jets $+E_{T}^{\text {miss }}$, etc. Standard Model processes (backgrounds, BG) which yield the same signatures must also be computed. Judicious cuts must be made to select signal events from BG. We invoke a multi-dimensional grid of cuts so as to optimize signal over BG in various regions of parameter space. By requiring signal $S$, for a given integrated luminosity (IL), to exceed $\max [5 \sigma, 5$ events, $0.2 \times B G]$, we can determine the reach of LHC for SUSY in the different multilepton channels.

The results from Ref. [13] are shown in Fig. 1. Even for $I L=0.1 \mathrm{fb}^{-1}$, the LHC7 reach extends far past Tevatron bounds: to $m_{\tilde{g}} \sim 800 \mathrm{GeV}$ for $m_{\tilde{q}} \simeq m_{\tilde{g}}$ ! For $1 \mathrm{fb}^{-1}$, then LHC reach is to over $m_{\tilde{g}}=1 \mathrm{TeV}$ for $m_{\tilde{g}}=m_{\tilde{q}}$, and to $m_{\tilde{g}} \sim 630 \mathrm{GeV}$ for $m_{\tilde{q}} \gg m_{\tilde{g}}$.

We will learn a LOT in 2011 about where SUSY is, or is not!

\section{LHC versus direct/indirect detection of neutralinos}

If neutralinos are the dark matter, then they may be detected via direct DM detection (DD) experiments such as Xenon-100 or LUX, or indirectly (IDD) via neutrino telescopes (searching 

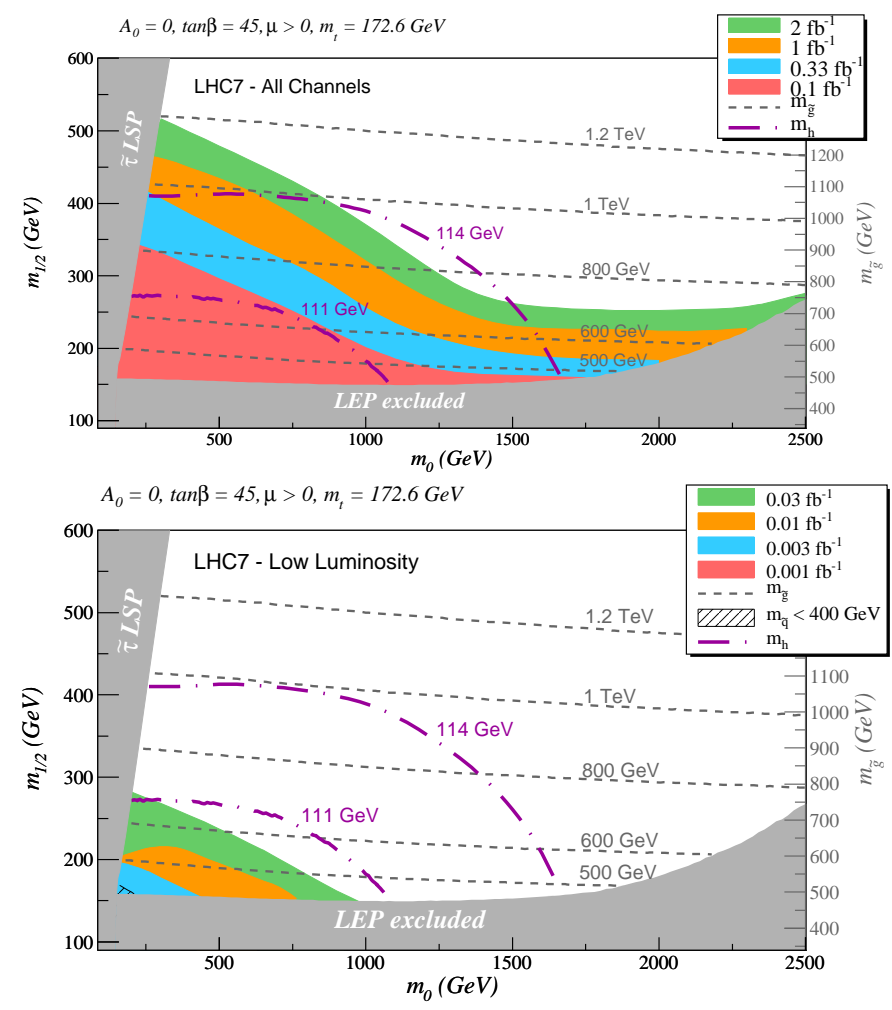

Figure 1: Upper frame: Reach of LHC7 for SUSY in the mSUGRA model with $\sqrt{s}=7 \mathrm{TeV}$ and integrated luminosity ranging from $100-2000 \mathrm{pb}^{-1}$. Lower frame: Reach of LHC7 for lower values of integrated luminosity.

for neutrinos from WIMP annihilation in the solar core), or by space-based antimatter (Pamela or AMS) or gamma ray detectors (Fermi-LAT).

We have calculated various DD and IDD signal rates in the mSUGRA model in Ref's [14] and [15], and compared to the reach of LHC14 and ILC500 and ILC1000. The results are shown in Fig. 2. Here, we see DD is largest when $m_{0}$ and $m_{1 / 2}$ are small (low squark masses enhance squark exchange) or in the hyperbolic branch/focus point region where the neutralino exists as mixed binohiggsino state and has a large Higgs exchange cross section. Since squarks are extremely heavy in the HB/FP region, this region has only limited coverage by LHC (up to $m_{\tilde{g}} \sim 1.4 \mathrm{TeV}$ for $100 \mathrm{fb}^{-1}$, while Xenon-100/LUX will likely cover the entire HB/FP region! For IDD, neutralino annihilation cross sections are also enhanced in the $\mathrm{HB} / \mathrm{FP}$ region, as well as in the $A$-resonance region.

\subsection{Unified explanation of current DD/IDD signals}

While I am on this topic, I note that a lot of excitement has been generated over the Pamela/ATIC/Fermi IDD signals, and also the DAMA/LIBRA, Cogent and CDMS DD signal events. I propose a unified explanation of these phenomena, by introducing a doublet of strongly interacting particles $(p, n)$, where $p$ has charge +1 and $n$ is neutral. Their putative mass is around the GeV scale. The $p$ s can be produced in various cosmic processes, and propagate a long ways: their flux should be much greater than that of positrons, and the gap between the two increases with energy. Since they have 


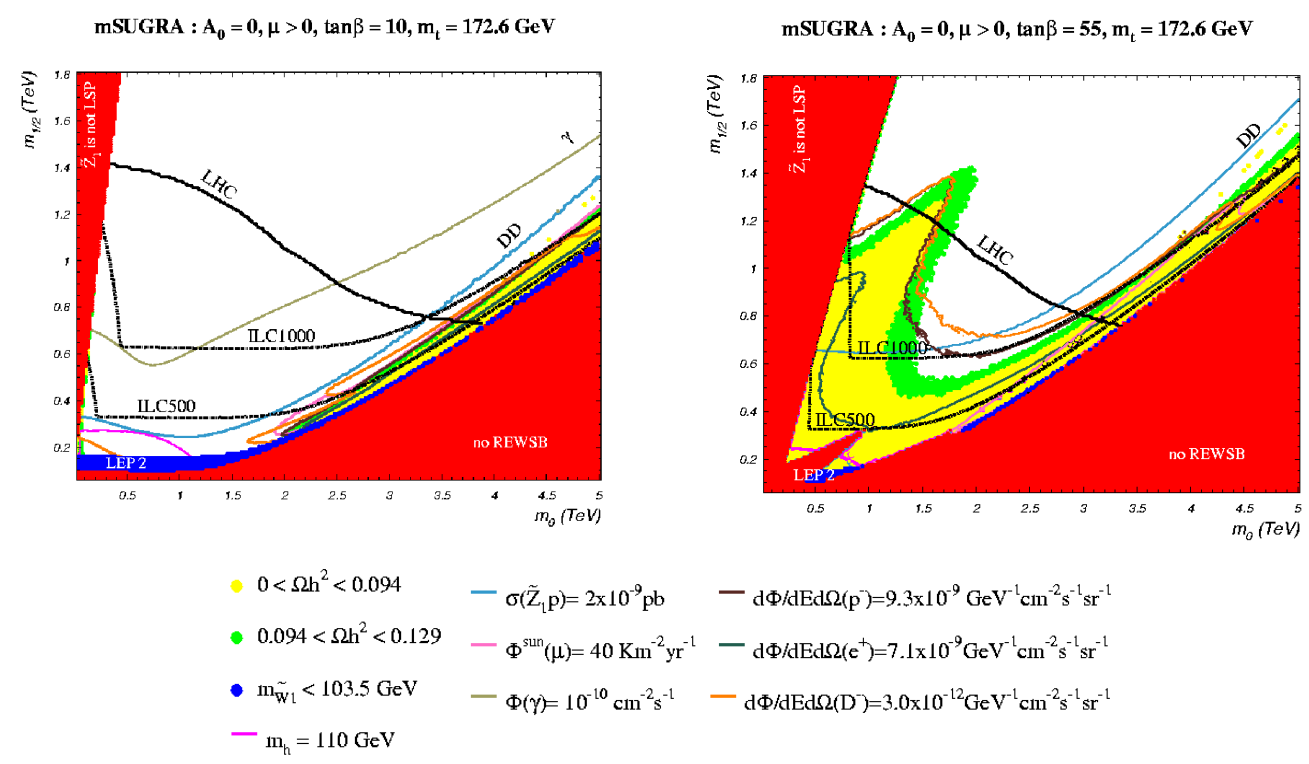

Figure 2: The projected reach of various colliders, direct and indirect dark matter search experiments in the $m_{0}$ vs. $m_{1 / 2}$ plane of the $m S U G R A$ model for $A_{0}=0, \mu>0, m_{t}=172.6 \mathrm{GeV}$ for $\tan \beta=10$ (left frame) and $\tan \beta=55$ (right frame). For the ID results, we have adopted the N03 DM halo density profile.

the same charge as $e^{+}$, they will occasionally be mistaken as a positron- and more often at higher energies.

In the case of $n$ particles, as emphasized by Ralston[16], these can be produced by muoninduced radioactivity even at great depths, with some seasonal dependence (due perhaps to density changes in the atmosphere in summer versus winter). Their cross sections for absorption and propagation vary wildly, and are ill-measured in the $\mathrm{keV}$ regime. Unless one invokes fiducialization by having a large mass detector with low BG, then some of these are expected to yield WIMP-like events.

\section{Well-tempered neutralinos (WTN)}

In Fig. 3, we show the spin-independent $\widetilde{Z}_{1} p$ cross section versus $m_{\widetilde{Z}_{1}}$ for a large number of one-parameter extensions of mSUGRA, where the GUT scale universality between matter scalar and Higgs scalar mass parameters, or between the three gaugino mass parameters is relaxed in a systematic way. The details of the various models are not essential for our present purpose, but may be found in Ref. [17]. In each such model, shown by a different colour on the plot, this additional parameter is adjusted so that the lightest neutralino (assumed to be the LSP) saturates the observed relic abundance of CDM. We also include the mSUGRA model. To make this plot, we randomly generated points in the parameter space for each model, and plotted it on the figure if all current collider constraints on sparticle masses are satisfied. We also show the sensitivity of current experiments together with projected sensitivity of proposed searches at superCDMS, Xenon-100, LUX, WARP and at a ton-sized noble liquid detector. The key feature to note is that while the various models have a branch where $\sigma_{\mathrm{SI}}\left(p \widetilde{Z}_{1}\right)$ falls off with $m_{\widetilde{Z}_{1}}$, there is another branch where 
this cross-section asymptotes to $\sim 10^{-8} \mathrm{pb}[17,18]$. This branch (which includes the HB/FP region of mSUGRA) includes many models with MHDM which easily accommodate the measured relic density via tempering of the neutralino's higgsino content[19]. In these cases, the spin-independent DD amplitude - which is mostly determined by the Higgs boson-higgsino-gaugino coupling - is large because the neutralino has both gaugino and higgsino components. The exciting thing is that the experiments currently being deployed- such as Xenon-100, LUX, WARP and superCDMS will have the necessary sensitivity to probe this entire class of models!

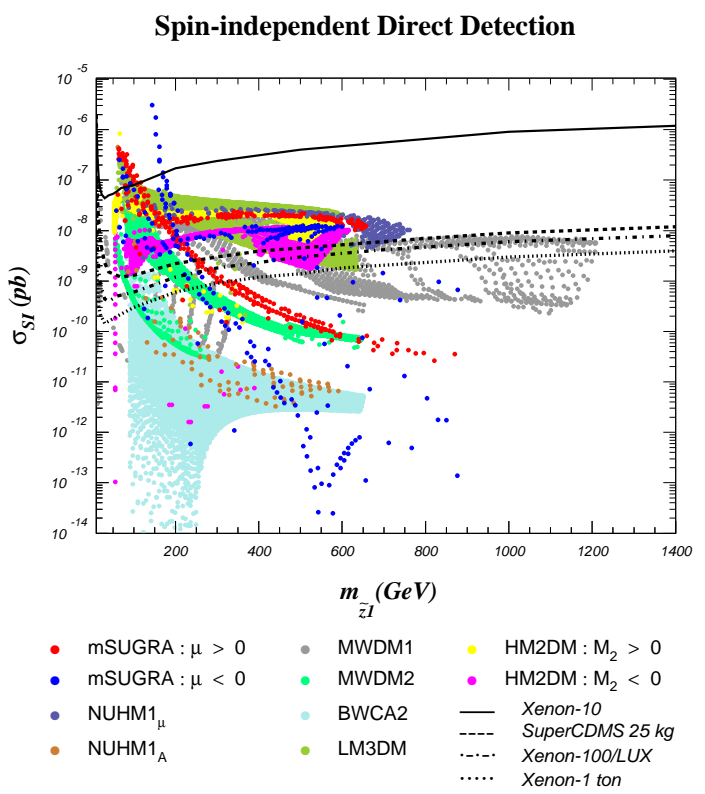

Figure 3: Predictions for $\sigma_{S I}\left(\widetilde{Z}_{1} p\right) v$ s. $m_{\widetilde{Z}_{1}}$, generally regarded as the figure of merit for direct detection experiments, in various models with $A_{0}=0$ and $m_{t}=171.4 \mathrm{GeV}$. The special parameter of each non-universal SUGRA model has been dialed to yield $\Omega_{\widetilde{Z}_{1}} h^{2} \simeq 0.11$. We fix $\tan \beta=10$ except for the mSUGRA model where we allow $\tan \beta=10,30,45,50,52$ and 55 . We also show the projected reach of selected direct detection experiments.

A key feature of the well-tempered neutralino models is that $\mu$ is comparable to gaugino mass $M_{1}$ at the weak scale so that we have a mixed bino-higgsino WIMP. This also implies the mass gap $m_{\widetilde{Z}_{2}}-m_{\widetilde{Z}_{1}}$ is in the range $\sim 20-90 \mathrm{GeV}$, which means in turn that $\widetilde{Z}_{2} \rightarrow \ell^{+} \ell^{-} \widetilde{Z}_{1}$ is a dominant decay mode, and should give rise to a visible mass edge with shape of $m\left(\ell^{+} \ell^{-}\right)$characteristic of a three-body decay at the LHC[17].

\subsection{Smoking gun LHC signature for low-mass SUSY or well-tempered neutralinos}

The decay $\widetilde{Z}_{2} \rightarrow \ell^{+} \ell^{-} \widetilde{Z}_{1}$ should occur with a large branching fraction in SUGRA models with gaugino mass unification if $m_{\tilde{g}} \lesssim 630 \mathrm{GeV}$, or in WTN models. The distribution rises to a sharp kinematic edge at $m_{\widetilde{Z}_{2}}-m_{\widetilde{Z}_{1}}$, which can lie between the photon and $Z$ poles[20]. This signature should be robust in OS-dilepton plus jets $+E_{T}^{\text {miss }}$ events at LHC, provided the above conditions hold, and that there is not accidental negative interference between slepton and $Z$ exchange in the three body decay amplitudes. 


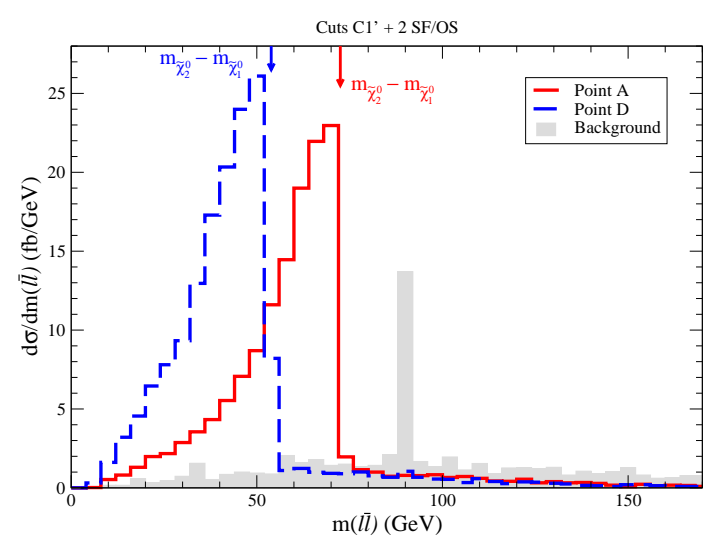

Figure 4: SF/OS dilepton invariant mass distribution after cuts $C_{1}^{\prime}$ from benchmark points A (full red line) and D (dashed blue line) along with SM backgrounds, for two Yukawa-unified benchmark points, from Ref. [21].

\section{Two problems for neutralino DM}

The idea of CDM consisting of neutralino WIMPs is a popular one. Much has been made lately of the so-called "WIMP miracle", wherein it is claimed that WIMPs have exactly the right properties to generate the observed abundance of dark matter, provided they undergo weak interactions and have weak scale masses. However, this argument applies better to a hypothetical left-hand neutrino with mass around the weak scale (perhaps in a fourth generation extension of the SM). Such massive left-hand neutrinos have long been ruled out because their direct detection cross sections are large.

When one plots the thermal neutralino relic density in the $m_{0} v s . m_{1 / 2}$ plane of the mSUGRA model, it is striking that almost all parameter space is ruled out, save for a few narrow regions: stau-coannihilation, $A$-resonance annihilation, HB/FP and the $h$-resonance. The relic density more typically in mSUGRA space is of order $\Omega_{\widetilde{Z}_{1}} h^{2} \sim 1-100$ : far too large. Rather high fine-tuning of parameters is needed to obtain the correct thermal abundance[22, 23]. One might object that this argument applies to mSUGRA, and other models with non-universal soft terms have more options to get the right relic density. In Ref. [24], we scanned over SUGRA models with 19 free parameters, and plotted the relic density. The result is shown in Fig. 5, where we have made a linear scan over 19 parameters, and required $m_{\widetilde{Z}_{1}}<500 \mathrm{GeV}$ to avoid too much electroweak fine-tuning. From the figure, it is clear that models with a bino-like neutralino should have $\Omega_{\widetilde{Z}_{1}} h^{2} \sim 1-1000$, while models with a wino-like or higgsino-like neutralino should have $\Omega_{\widetilde{Z}_{1}} h^{2} \sim 0.001-0.01$ : either far too much or far too little dark matter. The measured abundance falls exactly in the most improbable region, which requires high fine-tuning to get a well-tempered neutralino, or just the right sparticle mass combinations to get resonance or co-annihilation.

Another issue for the neutralino DM picture is the gravitino problem[25]. If gravitinos indeed set the mass scale for SUSY particles, and have weak scale masses, then they should be produced at rather high rates in the early universe, depending on the re-heat temperature $T_{R}$. The gravitinos will undergo late-time cascade decays to a neutralino LSP which will destroy the light element abundances built up in BBN, unless $T_{R} \lesssim 10^{5} \mathrm{GeV}$ [26]. If we accept $T_{R}<10^{5} \mathrm{GeV}$, it turns out 


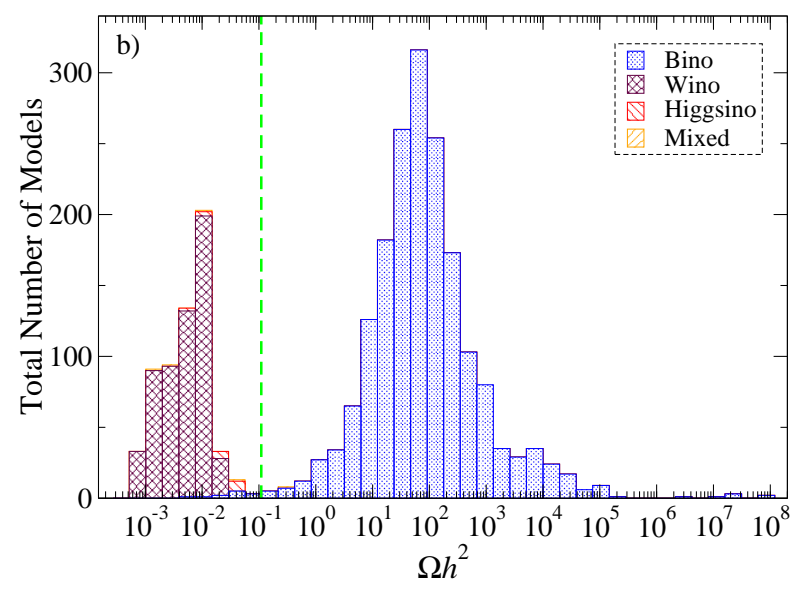

Figure 5: Projection of the number of models generated by a linear scan over SUGRA-19 parameters, versus neutralino relic density $\Omega_{\widetilde{Z}_{1}} h^{2}$. Models with mainly bino, wino, higgsino or a mixture are indicated by the various color and symbol choices. We require only models with $m_{\widetilde{Z}_{1}}<500 \mathrm{GeV}$ to avoid too large of fine-tuning in the SUSY parameters

this is not enough to support thermal leptogenesis which requires $T_{R} \gtrsim 2 \times 10^{9} \mathrm{GeV}$ [27]; and thermal leptogenesis seems favored by the emergent picture of neutrino masses via the see-saw mechanism. By requiring $m_{\tilde{G}} \gtrsim 5 \mathrm{TeV}$, the gravitino decays more quickly, and the bound[28] on $T_{R}$ goes up to $\sim 10^{8} \mathrm{GeV}$; this is enough to support at least non-thermal leptogenesis, wherein right-hand neutrinos are produced via other mechanisms, perhaps inflaton decay[29]. But then one should add into the abundance of neutralinos those that are produced from a high rate of gravitino production, which exacerbates the relic density problem for binos.

One might hypothesize the gravitino to be the LSP so it is stable and doesn't suffer late decays. But then if $\widetilde{Z}_{1}$ is NLSP, it will undergo late decays to gravitino plus hadrons, and re-introduce the gravitino problem.

Nature may be trying to tell us something here.

\section{Mixed axion/axino CDM}

Up to now, we have ignored the strong $C P$ problem. The Peccei-Quinn solution with an "invisible axion" [30, 31], is still the most attractive solution after over 30 years. In a SUSY context (the PQMSSM)[5], the axion is but one element of the axion/axino superfield, which includes along with the axion, the spin- $\frac{1}{2}, R$-parity-odd axino which may play the role of LSP, and the spin$0 R$-even saxion, which acquires a SUSY breaking mass. The axino mass is model dependent, and can lie anywhere from the $\mathrm{keV}-\mathrm{TeV}$ range. If $m_{\tilde{a}}<0.2 \mathrm{keV}$, then they could be produced in thermal equilibrium, but would constitute HDM[32]. If their mass is $\gtrsim 100 \mathrm{keV}$, then they can still be produced thermally (TP), but would constitute CDM[33]. Axino LSPs can also be produced from neutralino decays (NTP); since each neutralino decays to one axino, these non-thermally produced axinos inherit the neutralino number density, and reduce the neutralino abundance by a 
factor $m_{\tilde{a}} / m_{\widetilde{Z}_{1}}$. For $m_{\tilde{a}} \sim \mathrm{MeV}$ scale, this factor is tiny, and essentially wipes out the NTP axino abundance. The remaining abundance comes from a mixture of TP axinos and axions produced from vacuum mis-alignment. While the axino abundance decreases with PQ breaking scale $f_{a} / N$ due to decreasing coupling strength, the axion abundance increases with $f_{a} / N$, due to a likely larger initial axion field strength value.

The situation is shown in Fig. 6, where we plot the component contributions of mixed axion/axino CDM, requiring the total abundance $\Omega_{a \tilde{a}} h^{2}=0.11$. As $f_{a} / N$ increases, the TP axino contribution would like to decrease, but insisting on the measured total abundance requires an increased $T_{R}$ to compensate. In this way, in frame $b$ )., we see that $T_{R}$ can increase into the $10^{7}$ $\mathrm{GeV}$ range, sufficient enough to support non-thermal leptogenesis. Models with a very heavy gravitino[34], or the Asaka-Yanagida scenario[35] with $m$ (sparticle) $>m($ gravitino $)>m($ axino $)$ can even allow for $T_{R} \sim 10^{10}-10^{11} \mathrm{GeV}$, enough to support thermal leptogenesis! The highest $T_{R}$ values come when $f_{a} / N$ is quite large, $\sim 10^{12} \mathrm{GeV}$, when the mixed axion/axino dark matter is mainly axion $\mathrm{CDM}[36]$.

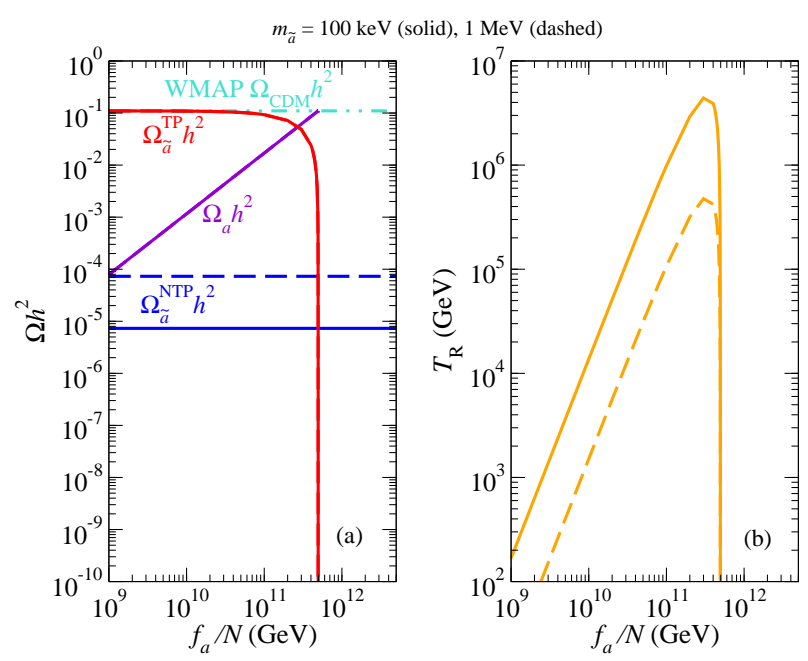

Figure 6: Axion and TP and NTP axino contributions to dark matter density for $m_{\tilde{a}}=100 \mathrm{keV}$ versus PQ breaking scale $f_{a} / N$.

For the mSUGRA model, we can adopt a rather large value of $f_{a} / N$ so that we get mainly axion CDM: $\Omega_{a} h^{2} \simeq 0.11$, with $\Omega_{\tilde{a}}^{T P} h^{2}=0.006$ and $\Omega_{\tilde{a}}^{N T P}=6 \times 10^{-6}$. Then in Fig. 7[36], we plot contours of $\log _{10} T_{R}$. We see that the regions with largest $\Omega_{\widetilde{Z}_{1}} h^{2}$ which would be severely excluded for $\widetilde{Z}_{1} \mathrm{DM}$ are now perfectly viable, and can yield $T_{R}$ over $10^{7} \mathrm{GeV}$ : the regions of mSUGRA space which are most highly disfavored by neutralino CDM are most favored by mainly axion CDM! A lesson: LHC searches for SUSY should not restrict themselves to so-called neutralino dark-matterallowed regions!

\section{Yukawa-unified SUSY and the LHC}

Armed with the idea of mixed axion/axino CDM, many compelling models which gave rise to far too much neutralino CDM can now be perfectly viable. One case is Yukawa unified SUSY, which is inspired by $S O(10)$ models with $t-b-\tau-v_{\tau}$ Yukawa coupling unification at $M_{G U T}$. 


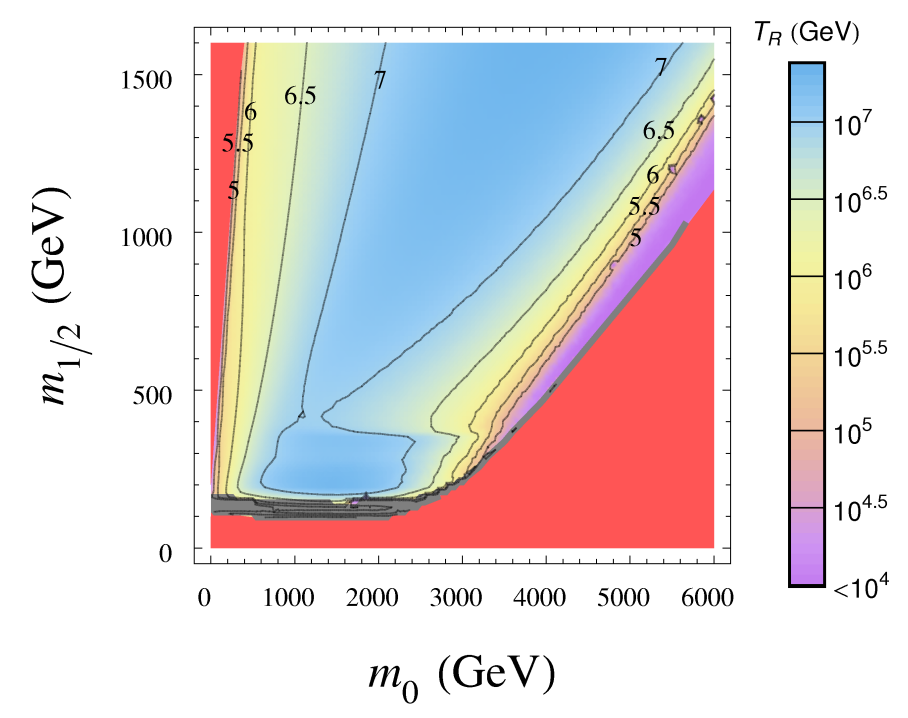

Figure 7: Contours of constant $T_{R}$ in the $m_{0}$ vs. $m_{1 / 2}$ plane for $A_{0}=0, \tan \beta=10$ and $\mu>0$. We assume $\Omega_{a} h^{2}=0.11$, and $\Omega_{\tilde{a}}^{T P} h^{2}=0.006$ and $\Omega_{\tilde{a}}^{N T P}=6 \times 10^{-6}$.

These models require $m_{16}$, the mass of all matter scalars, in the $\sim 10 \mathrm{TeV}$ range, while $m_{1 / 2}$ is very low[37]. One obtains a pure bino-like neutralino whose annihilation rate is severely suppressed by multi-TeV scalar exchange: $\Omega_{\widetilde{Z}_{1}} h^{2} \sim 10^{2}-10^{4}$. However, if we invoke mixed axion/axino CDM, the models become perfectly viable, with cases including mainly axion CDM as most favorable[38]. The prediction of Yukawa-unified SUSY is that $m_{\tilde{g}} \lesssim 500 \mathrm{GeV}$. LHC experiments will cover this range in 2011, thus ruling in or ruling out this class of models[21].

Another example is Effective SUSY, a model by Cohen, Kaplan and Nelson[39], which also involves multi-TeV scalars and too much neutralino CDM: this model is now perfectly viable with mixed axion/axino CDM[40].

\section{Conclusions}

My conclusions as a bullet list:

- The role of LHC in dark matter searches: produce matter states associated with the dark matter, which cascade decay into DM states. The usual signature is multi-jet plus multilepton plus $E_{T}^{\text {miss }}$ events, which might be accompanied by quasi-stable particle states.

- In the case of WIMP dark matter, additional signals from direct and indirect detection experiments should provide complementary information.

- Th Xe-100 experiment will soon test SUSY models with well-tempered neutralinos, which have DD cross sections at $\sim 10^{-8} \mathrm{pb}$; these models should exhibit a $m\left(\ell^{+} \ell^{-}\right)$mass edge below $90 \mathrm{GeV}$ at LHC.

- Neutralinos suffer from tending to predict too big (bino-like) or too small (wino or higgsinolike) a relic density. They also suffer from the gravitino problem. 
- Ditto for gravitinos

- Mixed axion/axino CDM avoids both these isues, plus includes a solution to the strong CP problem

- Very compelling models such as $S O(10)$-inspired Yukawa-unified SUSY yield far too much neutralino dark matter. However, these models are perfectly viable with mixed axion/axino dark matter. Since they predict $m_{\tilde{g}} \lesssim 500 \mathrm{GeV}$ (to allow for Yukawa coupling unification), they will be ruled in or out by LHC in 2011.

Acknowledgements: I thank V. Barger, A. Box, S. Kraml, A. Lessa, A. Mustafayev, E. K. Park, S. Sekmen, H. Summy, and X. Tata for fruitful collaborations, and I thank the organizers of IDM2010 for creating an excellent conference!

\section{References}

[1] H. Baer and X. Tata, Weak Scale Supersymmetry: From Superfields to Scattering Events, (Cambridge University Press, 2006).

[2] R. Peccei and H. Quinn, Phys. Rev. Lett. 38 (1977) 1440 and Phys. Rev. D 16 (1977) 1791; S. Weinberg, Phys. Rev. Lett. 40 (1978) 223; F. Wilczek, Phys. Rev. Lett. 40 (1978) 279.

[3] For a recent review, see J. E. Kim and G. Carosi, arXiv:0807.3125.

[4] L. F. Abbott and P. Sikivie, Phys. Lett. B 120 (1983) 133; J. Preskill, M. Wise and F. Wilczek, Phys. Lett. B 120 (1983) 127; M. Dine and W. Fischler, Phys. Lett. B 120 (1983) 137; M. Turner, Phys. Rev. D 33 (1986) 889; L. Visinelli and P. Gondolo, Phys. Rev. D 80 (2009) 035024.

[5] H. P. Nilles and S. Raby, Nucl. Phys. B 198 (1982) 102; J. E. Kim, Phys. Lett. B 136 (1984) 378; J. E. Kim and H. P. Nilles, Phys. Lett. B 138 (1984) 150.

[6] H. Baer, R. Dermisek, S. Rajagopalan and H. Summy, JCAP1007 (2010) 014.

[7] For a review, see R. Arnowitt and P. Nath, arXiv:0912.2273 (2009).

[8] H. Baer, C. H. Chen, R. Munroe, F. Paige and X. Tata, Phys. Rev. D 51 (1995) 1046; H. Baer, J. Ferrandis, S. Kraml and W. Porod, Phys. Rev. D 73 (2006) 015010.

[9] F. Paige, S. Protopopescu, H. Baer and X. Tata, hep-ph/0312045.

[10] H. Baer, C. Balazs and A.Belyaev, J. High Energy Phys. 0203 (2002) 042.

[11] H. Baer, J. Ellis, G. Gelmini, D. V. Nanopoulos and X. Tata, Phys. Lett. B 161 (1985) 175; G. Gamberini, Z. Physik C 30 (1986) 605; H. Baer, V. Barger, D. Karatas and X. Tata, Phys. Rev. D 36 (1987) 96; H. Baer, X. Tata and J. Woodside, Phys. Rev. D 42 (1990) 1568.

[12] H. Baer, X. Tata and J. Woodside, Phys. Rev. D 45 (1992) 142; H. Baer, C. H. Chen, F. Paige and X. Tata, Phys. Rev. D 52 (1995) 2746 and Phys. Rev. 55 (1996) 6241; H. Baer, C. H. Chen, M. Drees, F. Paige and X. Tata, Phys. Rev. D 59 (1999) 055014 H. Baer, C. Balázs, A. Belyaev, T. Krupovnickas and X. Tata, J. High Energy Phys. 0306 (2003) 054.

[13] H. Baer, V. Barger, A. Lessa and X. Tata, J. High Energy Phys. 1006 (2010) 102

[14] H. Baer, A. Belyaev, T. Krupovnickas and J. O’Farrill, JCAP 0408 (2004) 005. 
[15] H. Baer, E. K. Park and X. Tata, New Jou. Phys.11 (2009) 105024.

[16] J. Ralston, arXiv:1006.5255 (2010).

[17] H. Baer, A. Mustafayev, E. Park and X. Tata, JCAP 0701, 017 (2007); H. Baer, A. Mustafayev, E. Park and X. Tata, J. High Energy Phys. 0805 (2008) 058.

[18] D. Feldman, Z. Liu and P. Nath, Phys. Lett. B 662 (2008) 190.

[19] N. Arkani-Hamed, A. Delgado and G. F. Giudice, Nucl. Phys. B 741 (2006) 108.

[20] H. Baer, K. Hagiwara and X. Tata, Phys. Rev. D 35 (1987) 1598; H. Baer, D. Dzialo-Karatas and X. Tata, Phys. Rev. D 42 (1990) 2259; H. Baer, C. Kao and X. Tata, Phys. Rev. D 48 (1993) 5175; I. Hinchliffe, F. Paige, M. Shapiro, J. Soderqvist and W. Yao, Phys. Rev. D 55 (1997) 5520.

[21] H. Baer, S. Kraml, S. Sekmen and H. Summy, J. High Energy Phys. 0810 (2008) 079; H. Baer, S. Kraml, A. Lessa and S. Sekmen, J. High Energy Phys. 0911 (2010) 055;

[22] J. Ellis and K. Olive, Phys. Lett. B 514 (2001) 114.

[23] H. Baer and A. Box, Eur. Phys. J. C 68 (2010) 523.

[24] H. Baer, A. Box and H. Summy, arXiv:1005.2215 (2010)

[25] S. Weinberg, Phys. Rev. Lett. 48 (1982) 1303.

[26] M. Khlopov and A. Linde, Phys. Lett. B 138 (1984) 265.

[27] W. Buchmüller, P. Di Bari and M. Plumacher, Nucl. Phys. B 643 (2002) 367 and Erratum-ibid,B793 (2008) 362; Annals Phys. 315 (2005) 305 and New Jou. Phys.6 (2004) 105.

[28] M. Kawasaki, K. Kohri, T. Moroi and A. Yotsuyanagi, arXiv:0804.3745 (2008).

[29] G. Lazarides and Q. Shafi, Phys. Lett. B 258 (1991) 305; K. Kumekawa, T. Moroi and T. Yanagida, Prog. Theor. Phys. 92 (1994) 437; T. Asaka, K. Hamaguchi, M. Kawasaki and T. Yanagida, Phys. Lett. B 464 (1999) 12.

[30] J. E. Kim, Phys. Rev. Lett. 43 (1979) 103; M. A. Shifman, A. Vainstein and V. I. Zakharov, Nucl. Phys. B 166 (1980) 493.

[31] M. Dine, W. Fischler and M. Srednicki, Phys. Lett. B 104 (1981) 199; A. P. Zhitnitskii, Sov. J. Nucl. 31 (1980) 260.

[32] K. Rajagopal, M. Turner and F. Wilczek, Nucl. Phys. B 358 (1991) 447.

[33] L. Covi, J. E. Kim and L. Roszkowski, Phys. Rev. Lett. 82 (1999) 4180; L. Covi, H. B. Kim, J. E. Kim and L. Roszkowski, J. High Energy Phys. 0105 (2001) 033.

[34] H. Baer, S. Kraml, A. Lessa and S. Sekmen, JCAP () .

[35] T. Asaka and T. Yanagida, Phys. Lett. B 494 (2000) 297; H. Baer, S. Kraml, A. Lessa and S. Sekmen, to appear.

[36] H. Baer, A. Box and H. Summy, J. High Energy Phys. 0908 (2009) 080.

[37] D. Auto, H. Baer, C. Balazs, A. Belyaev, J. Ferrandis and X. Tata, J. High Energy Phys. 0306 (2003) 023; H. Baer, S. Kraml, S. Sekmen and H. Summy, J. High Energy Phys. 0803 (2008) 056.

[38] H. Baer, M. Haider, S. Kraml, S. Sekmen and H. Summy, JCAP0902 (2009) 002.

[39] A. Cohen, D. Kaplan and A. Nelson, Phys. Lett. B 388 (1996) 588.

[40] H. Baer, S. Kraml, A. Lessa, S. Sekmen and X. Tata, J. High Energy Phys. 1010 (2010) 018. 